\title{
Field performance assessment of the ADVANCE-F automatic steering control vehicle
}

\author{
Tang-Hsien Chang* \\ Department of Civil Engineering, National Taiwan University, P.O. Box 7-876, Taipei 10617, Taiwan, ROC
}

Received 11 July 2002; accepted 27 June 2003

\begin{abstract}
This paper describes the field verification of the ADVANCE-F's lateral controller for vehicle steering automation and hands-free lane keeping. The controller steers an equipped vehicle along a specially marked lane without cross-over lane markings. Testing results give preliminary verification that controller performance is stable and has robust capability on meandering roads. The results also reveal that the automatic steering mode can overcome the effects of unbalanced loading, missing alignment, and a distorted positioning of the photosensors that are provided for lateral guidance of the vehicle. A smooth transition process from the manual to the automatic mode is proposed to avoid abnormal and over-shooting situations.
\end{abstract}

(C) 2003 Elsevier Ltd. All rights reserved.

Keywords: ITS; Intelligent transport systems; Automatic vehicle control systems; Vehicle lateral controller; Automatic steering systems

\section{Introduction}

According to the proceedings of the world congress on ITS from 1994 to 2002, many institutes and vehicle manufacturers engaged in the study of Advanced Vehicle Control Systems (AVCS). AVCS maneuvers the equipped vehicle more precisely and stably when car-following and lane-keeping, improving highway capacity and traffic safety (Fenton, 1979; Ioannou, Chien, \& Hauser, 1992; Rao, Varaiya, \& Eskafi, 1993). ADVANCE-F is a project attempting to elevate Taiwan's highway traffic quality and transportation quantity by developing an affordable AVCS (Chang, 1993, 1995). This study describes and assesses the ADVANCE-F automatic steering mode. The automatic steering mode keeps the equipped vehicle running along a lane's specially marked centerline without manual handling. Fig. 1 describes the system configuration and component relationships, and highlights that the special material markings embedded in the selected lane is the only public facility required. The width of the markings is $0.0254 \mathrm{~m}(1 \mathrm{in})$.

*Tel.: + 886-223-625-920; fax: + 886-223-639-990

E-mail address: thchang@ce.ntu.edu.tw (T.-H. Chang).
In-vehicle equipment includes the sensors, computer, steering controller and stepping motor, etc. There are two sets of photosensors mounted in parallel beneath the front and rear bumpers of the vehicle. Each set has 15 sensor units, equipped at equal, symmetrically distributed intervals no greater than $0.05 \mathrm{~m}$, that emit infrared to detect the guidance markings on the roadway. Any sensor capturing the marking will transmit a signal to the on-board computer (OBC). The computer can calculate the vehicle's yaw angle and lateral deviation as well as estimate their rates of variation in real time, based on the front and rear sensor signals. The vehicle speed and steering angle will also be measured from time to time. The designed control program allows the computer to trigger the motor driver to engage the stepping motor and keep the vehicle in and along the lane with stability.

The system has been subjected to the field test. The following paragraphs briefly describe the ADVANCE-F system's steering control methodology, followed by discussion of the field test and a comparison between human handling and the automatic driving mode. Finally, a control algorithm for switching-in and switching-out of the automatic steering system is presented since the transition control from manual to automatic driving is critical to avoid serious danger. 


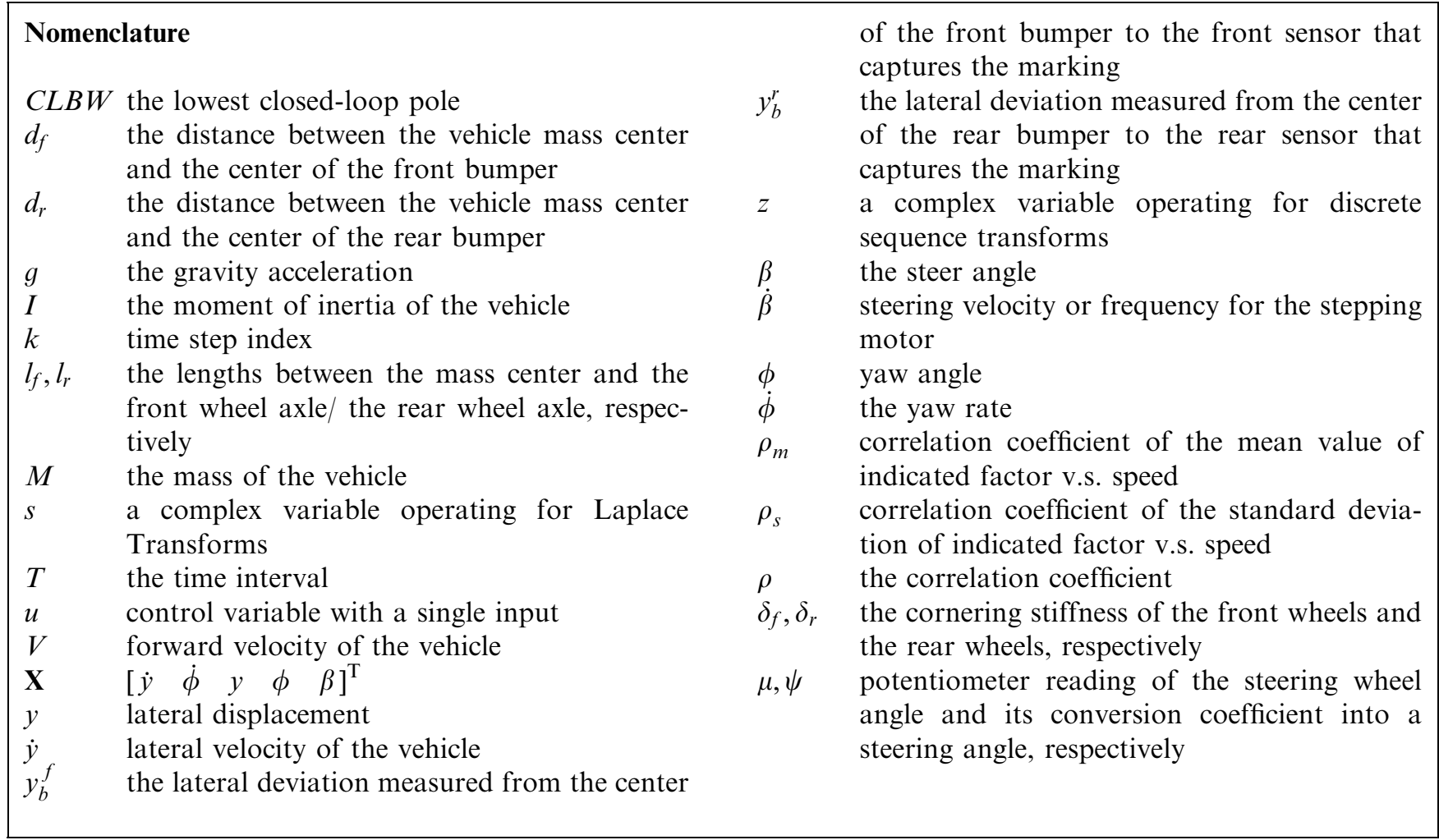

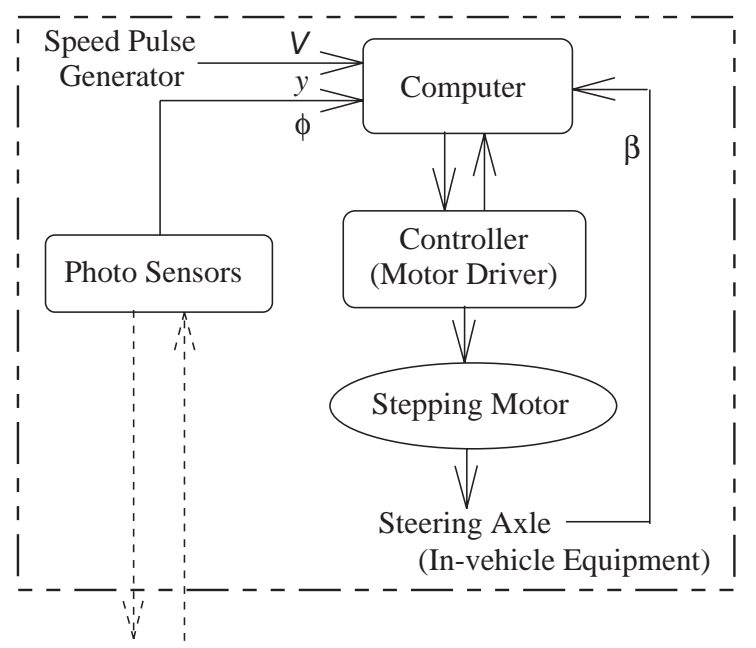

Marking (Roadway Infrastructure)

Fig. 1. ADVANCE-F's lateral control frame.

\section{Lateral controller}

Firstly, a major task is to devise a core controller to completely handle the vehicle track within an acceptably small deviation at any time and in any situation. The author has used the optimization approach to devise such a controller which concentrates on minimizing a given performance function that satisfies several parameters. Fig. 2 shows the features of the ADVANCE-F vehicle's lateral controller. $\mathbf{X}$ is the system column vector with element lateral velocity $\dot{y}$, yaw rate $\dot{\phi}$, lateral displacement $y$, yaw angle $\phi$, and steer angle $\beta$. $\mathbf{Y}$ is the output column vector with measured elements, $y, \phi$, and $\beta$. Control variable, $u$, is denoted as the steering rate $\dot{\beta}$. A, $\mathbf{B}$, and $\mathbf{C}$ are parameter matrices. An observer for estimating $\dot{y}$ and $\dot{\phi}$ was built to reduce the equipment cost. The control law from the feedback regulator has been derived by Chang (1996a):

$u=\left(-\mathbf{G}_{0} \mathbf{G}_{e}\right) \hat{\mathbf{X}}+\left(-\mathbf{E}^{\mathrm{T}}\right) \mathbf{U}$,

where

$\hat{\mathbf{X}}=\left[\mathbf{x}_{e} \mathbf{Y}\right]^{\mathrm{T}}$

and

$\mathrm{x}_{e} \underline{\Delta} \operatorname{est}\left(\left[\begin{array}{l}\dot{y} \\ \dot{\phi}\end{array}\right]\right)$.

The regulator $\mathbf{U}$ and the estimator $\mathbf{x}_{e}$ should satisfy

$$
\begin{aligned}
\dot{\mathbf{U}}= & (-\Lambda) \mathbf{U}+\left(\Phi \mathbf{G}_{e}\right) \hat{\mathbf{X}} \\
\dot{\mathbf{x}}_{e}= & \left(\mathbf{A}_{11}-K_{e} \mathbf{A}_{21}\right) \mathbf{x}_{e}+\left(\mathbf{B}_{1}-K_{e} \mathbf{B}_{2}\right)\left(\mathbf{A}_{12}-K_{e} \mathbf{A}_{22}\right) \\
& \left.+\left(\mathbf{A}_{11}-K_{e} \mathbf{A}_{21}\right) K_{e}\right]\left[\begin{array}{c}
u \\
\mathbf{Y}
\end{array}\right],
\end{aligned}
$$

where

$\mathbf{G}_{0}=\left[\begin{array}{lllll}\tilde{g}_{1} & \tilde{g}_{2} & \tilde{g}_{3} & \tilde{g}_{4} & \tilde{g}_{5}\end{array}\right]$, 
$\mathbf{G}_{e}=\left[\begin{array}{ccccc}1 & 0 & k 1(V) & k 2(V) & 0 \\ 0 & 1 & k 3(V) & k 4(V) & 0 \\ 0 & 0 & 1 & 0 & 0 \\ 0 & 0 & 0 & 1 & 0 \\ 0 & 0 & 0 & 0 & 1\end{array}\right]$

$K_{e}=\left[\begin{array}{lll}k 1(V) & k 2(V) & 0 \\ k 3(V) & k 4(V) & 0\end{array}\right]$,

$\mathbf{E}=\left[\begin{array}{lllll}1 & 1 & 1 & 1 & 1\end{array}\right]^{\mathrm{T}}$,

$\Lambda=\left[\begin{array}{cccccc}1 / \lambda_{1} & 0 & 0 & 0 & 0 & 0 \\ 0 & 1 / \lambda_{2} & 0 & 0 & 0 & 0 \\ 0 & 0 & 1 / \lambda_{3} & 0 & 0 & 0 \\ 0 & 0 & 0 & 1 / \lambda_{4} & 0 & 0 \\ 0 & 0 & 0 & 0 & 1 / \lambda_{5} & 0 \\ 0 & 0 & 0 & 0 & 0 & 1 / \lambda_{6}\end{array}\right]$,

$\Phi=\left[\begin{array}{ccccc}\tilde{g}_{6} / \lambda_{1} & 0 & 0 & 0 & 0 \\ 0 & \tilde{g}_{7} / \lambda_{2} & 0 & 0 & 0 \\ 0 & 0 & \tilde{g}_{8} / \lambda_{3} & 0 & 0 \\ 0 & 0 & 0 & \tilde{g}_{9} / \lambda_{4} & 0 \\ 0 & 0 & 0 & 0 & \tilde{g}_{10} / \lambda_{5} \\ \tilde{g}_{11} a_{11} / \lambda_{6} & \tilde{g}_{11} a_{12} / \lambda_{6} & 0 & \tilde{g}_{11} a_{14} / \lambda_{6} & \tilde{g}_{11} a_{15} / \lambda_{6}\end{array}\right]$,

$\mathbf{A}_{11}=\left[\begin{array}{ll}a_{11} & a_{12} \\ a_{21} & a_{22}\end{array}\right], \quad \mathbf{A}_{12}=\left[\begin{array}{lll}0 & a_{14} & a_{15} \\ 0 & a_{24} & a_{25}\end{array}\right], \quad \mathbf{B}_{1}=\left[\begin{array}{l}0 \\ 0\end{array}\right]$,

$\mathbf{A}_{21}=\left[\begin{array}{ll}1 & 0 \\ 0 & 1 \\ 0 & 0\end{array}\right], \quad \mathbf{A}_{22}=\left[\begin{array}{lll}0 & 0 & 0 \\ 0 & 0 & 0 \\ 0 & 0 & 0\end{array}\right], \quad \mathbf{B}_{2}=\left[\begin{array}{l}0 \\ 0 \\ 1\end{array}\right]$,

$a_{11}=-2\left(\delta_{f}+\delta_{r}\right) / M V, \quad a_{21}=-2\left(\delta_{f} \cdot l_{f}-\delta_{r} \cdot l_{r}\right) / I V$,

$a_{12}=-2\left(\delta_{f} \cdot l_{f}-\delta_{r} \cdot l_{r}\right) / M V, \quad a_{22}=-2\left(\delta_{f} \cdot l_{f}^{2}+\delta_{r} \cdot l_{r}^{2}\right) / I V$,

$a_{14}=2\left(\delta_{f}+\delta_{r}\right) / M, \quad a_{24}=2\left(\delta_{f} \cdot l_{f}-\delta_{r} \cdot l_{r}\right) / I$,

$a_{15}=2 \delta_{f} / M, \quad a_{25}=2 \delta_{f} \cdot l_{f} / I$

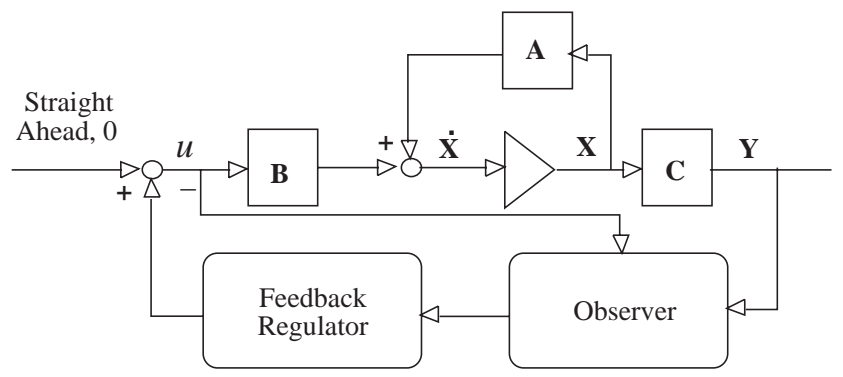

Fig. 2. Feature of the lateral controller. $\tilde{g}_{i}, \quad i=1,2, \ldots, 11, \lambda_{j}, j=1,2, \ldots, 6$, and $k 1(V), k 2(V)$, $k 3(V), k 4(V)$ are parameters varied on different levels of velocity as illustrated in Chang (1996a).

For implementation, take bilinear C2D transformation (continuous state to discrete state) for Eqs. (1), (4) and (5) by the Tustin's method (Vegte, 1994) as

$s=\frac{2}{T}\left(\frac{z-1}{z+1}\right)$

where $s$ is a complex variable operating for the Laplace transformation, $T$ is the time interval and $z$ is also a complex variable operating for a discrete sequence in the geometric transformation (a $z$-transform). The time interval is determined by choosing an optimal cycle for the execution of the control command (Fenton, 1988):

$T \leqslant \frac{1}{10 C L B W}$,

where $C L B W$ denotes the lowest closed-loop pole. Herein, the time $T$ is selected as $30 \mathrm{~ms}$, which is well within the limit. Then we have, in a state $k, k=$ $0,1,2, \ldots$, the form

$u(k)=\left(-\mathbf{G}_{0} \mathbf{G}_{e}\right)\left[\begin{array}{c}\mathrm{x}_{\mathrm{e}}(k) \\ \mathbf{Y}(k)\end{array}\right]+\left(-\mathbf{E}^{\mathrm{T}}\right) \mathbf{U}(k)$,

$\mathbf{U}(k)=\hat{\Lambda} \mathbf{U}(k-1)+\tilde{\Phi}\left[\begin{array}{c}\mathrm{x}_{\mathrm{e}}(k-1) \\ \mathbf{Y}(k-1)\end{array}\right]$,

$\mathrm{x}_{e}(k)=\tilde{\mathbf{A}}_{e} \mathrm{x}_{e}(k-1)+\tilde{\mathbf{B}}_{e}\left[\begin{array}{c}u(k-1) \\ \mathbf{Y}(k-1)\end{array}\right]$.

Expanding the elements of the above three matrix equations, which yield to the following nine algebraic equations in terms of 28 parameters of $\xi_{i}, i=1$, $2, \ldots, 28$ :

$$
\begin{aligned}
u(k)= & \xi_{1} x_{e 1}(k)+\xi_{2} x_{e 2}(k)+\xi_{3} y_{1}(k)+\xi_{4} y_{2}(k)+\xi_{5} y_{3}(k) \\
& -v_{1}(k)-v_{2}(k)-v_{3}(k)-v_{4}(k)-v_{5}(k)-v_{6}(k),
\end{aligned}
$$

$v_{1}(k)=\xi_{9} x_{e 1}(k-1)+\xi_{10} y_{1}(k-1)+\xi_{11} y_{2}(k-1)$,

$$
\begin{aligned}
v_{2}(k)= & \xi_{6} v_{2}(k-1)+\xi_{12} x_{e 2}(k-1) \\
& +\xi_{13} y_{1}(k-1)+\xi_{14} y_{2}(k-1),
\end{aligned}
$$

$v_{3}(k)=\xi_{15} y_{1}(k-1)$ 
$v_{4}(k)=\xi_{16} y_{2}(k-1)$

$v_{5}(k)=\xi_{7} v_{5}(k-1)+\xi_{17} y_{3}(k-1)$,

$v_{6}(k)=\xi_{8} v_{6}(k-1)+\xi_{18} x_{e 1}(k-1)+\xi_{19} x_{e 2}(k-1)$

$$
+\xi_{20} y_{1}(k-1)+\xi_{21} y_{2}(k-1)+\xi_{22} y_{3}(k-1),
$$

$$
\begin{aligned}
& x_{e 1}(k)=\xi_{23} y_{1}(k-1)+\xi_{24} y_{2}(k-1)+\xi_{25} y_{3}(k-1), \\
& x_{e 2}=\xi_{26} y_{1}(k-1)+\xi_{27} y_{2}(k-1)+\xi_{28} y_{3}(k-1) .
\end{aligned}
$$

Corresponding to the test car, $M=1120 \mathrm{~kg}, I=$ $2040 \mathrm{~kg} \mathrm{~m}^{2}, l_{f}=1.10 \mathrm{~m}, l_{r}=1.55 \mathrm{~m}, \quad \delta_{f}=48000 \mathrm{~N} / \mathrm{rad}$ and $\delta_{r}=42000 \mathrm{~N} / \mathrm{rad}$, parameters, $\xi_{i}, i=1,2, \ldots, 28$ can be calculated when relevant to $\mathbf{G}_{0}, \mathbf{G}_{e}, \mathbf{E}, \tilde{\Lambda}, \tilde{\Phi}, \tilde{\mathbf{A}}_{e}, \tilde{\mathbf{B}}_{e}$ and with respect to different speeds, $V=1,2, \ldots$ $120 \mathrm{kph}$. Also, since $y_{i}(k), i=1,2,3$, are three components of vector $\mathbf{Y}(k)$, these are measured by two sets of sensors which are mounted beneath the front and rear bumpers, and a potentiometer installed beside the steer axle. In detailed expression, at a certain state,

$$
\mathbf{Y}=\left[\begin{array}{l}
y_{1} \\
y_{2} \\
y_{3}
\end{array}\right]=\left[\begin{array}{l}
y \\
\phi \\
\beta
\end{array}\right]=\left[\begin{array}{ccc}
\frac{d_{r}}{d_{f}+d_{r}} & \frac{d_{f}}{d_{f}+d_{r}} & 0 \\
\frac{1}{d_{f}+d_{r}} & \frac{-1}{d_{f}+d_{r}} & 0 \\
0 & 0 & \mu
\end{array}\right]\left[\begin{array}{c}
y_{b}^{f} \\
y_{b}^{r} \\
\psi
\end{array}\right],
$$

where $d_{f}$ is the distance between the vehicle mass center and the center of the front bumper, $d_{r}$ is the distance between the vehicle mass center and the center of the rear bumper, $y_{b}^{f}$ is the lateral deviation measured from the center of the front bumper to the front sensor that captures the marking, $y_{b}^{r}$ is the lateral deviation measured from the center of the rear bumper to the rear sensor that captures the marking. $\mu, \psi$ are the steering wheel angle read from the potentiometer and its conversion coefficient into a steering angle, respectively.

The control input from Eq. (11) can be obtained by substituting the information of Eq. (20) into Eqs. (12)-(19), and then used to accomplish the vehicle lateral control.

\section{Human drivers versus the automatic driving mode}

The eigenvalues of the state matrix of the entire system equation are entirely negative (Chang, 1996a). This fact demonstrates that the model is stable (Kuo, 1991). The model has withstood the noisy rejections of Chang's simulations (Chang, 1996a), including side wind disturbances and curve transitions. However, whether such stability is accepted or not by a driver is measured in accordance with the following task.

A comparison between the manual and automatic modes in the same driving circumstances was made by presenting the field performance of the automatic steering system. The "visual direction" of an ADVANCE-F vehicle is quite different from a human driver's because, unlike humans who can watch hundreds of meters ahead when driving, the ADVANCE-F's vehicle uses photosensors mounted under the front and rear bumpers that look down to detect guidance markings along the road. The decision and steering actuation time are among the most important factors affecting the vehicle's dynamics. The response time should be as rapid as possible. Fortunately, the photosensor response time is much shorter than a human's. Whether the ADVANCE-F's performance is acceptable to passengers can be judged by a comparison of the outputs from human driving and automatic driving.

The test was conducted in a near straight lane, $450 \mathrm{~m}$ long. The equipped vehicle was handled by an experienced driver over four runs with speeds of $20 \mathrm{kph}$, $30 \mathrm{kph}, 40 \mathrm{kph}$ and $50 \mathrm{kph}$, respectively. Based on the time series records from the mounted front/rear sensors and the steering wheel, the vehicle's lateral displacement, yaw angle, and steering angle are measured. Table 1 shows the analytical outputs from the time series data collected. The table indicates the following.

(1) Lateral displacement: According to Table 1, the lateral displacement has a low correlation $\left(\rho_{m}=-0.3782\right)$ to vehicle speed. This means an experienced/normal driver can track the lane within reasonable speeds. However, the standard deviation is reversed and has a high positive correlation $\left(\rho_{s}=0.9742\right)$ to vehicle speed. Such a standard deviation becomes larger as speed increases, indicating that humans have more difficulty handling a high-speed vehicle than a low-speed vehicle. Consequently, the lane and the lateral clearance of the high-speed road must be wider than that of a lowspeed road. This inference matches current highway design criteria.

(2) Yaw angle variation: The yaw angle variations are always positive, but all the mean values of the lateral displacement are negative. This phenomenon reveals that the testing vehicle has a bias on body dynamics, perhaps because of unbalanced loading, missing alignment, or photosensor disposition or distortion. $\left(\rho_{m}=-0.5211, \rho_{s}=0.7568\right)$

(3) Steering angle variation: The positive magnitude of the steering angle confirms that the testing vehicle's attribute is definitely biased as a result of unbalanced loading, missing alignment, or photosensor disposition or distortion. $\left(\rho_{m}=0.4765\right.$, $\left.\rho_{s}=-0.043\right)$. 
Table 1

Results of the manual mode

\begin{tabular}{|c|c|c|c|c|c|}
\hline & & \multicolumn{4}{|l|}{ Speeds } \\
\hline & & $20 \mathrm{kph}$ & $30 \mathrm{kph}$ & $40 \mathrm{kph}$ & $50 \mathrm{kph}$ \\
\hline \multirow[t]{2}{*}{ Lateral displacement } & Mean $(\mathrm{cm})$ & -4.085 & -12.861 & -0.247 & -15.221 \\
\hline & Standard deviation $(\hat{\sigma})$ & 1.530 & 2.366 & 2.772 & 3.102 \\
\hline \multirow[t]{2}{*}{ Yaw angle variation } & Mean (deg) & 0.391 & 0.401 & 0.507 & 0.157 \\
\hline & Standard deviation $(\hat{\sigma})$ & 0.241 & 0.270 & 0.459 & 0.373 \\
\hline \multirow[t]{2}{*}{ Steer angle variation } & Mean(deg) & 2.064 & 8.687 & 3.646 & 7.631 \\
\hline & Standard deviation $(\hat{\sigma})$ & 3.091 & 3.275 & 2.032 & 3.435 \\
\hline
\end{tabular}

Table 2

Results of the automatic mode

\begin{tabular}{|c|c|c|c|c|c|}
\hline & & \multicolumn{4}{|l|}{ Speeds } \\
\hline & & $20 \mathrm{kph}$ & $30 \mathrm{kph}$ & $40 \mathrm{kph}$ & $50 \mathrm{kph}$ \\
\hline \multirow[t]{2}{*}{ Lateral displacement } & Mean $(\mathrm{cm})$ & -4.496 & -3.911 & -1.080 & 0.56 \\
\hline & Standard deviation $(\hat{\sigma})$ & 1.897 & 1.587 & 1.338 & 0.95 \\
\hline \multirow[t]{2}{*}{ Yaw angle variation } & Mean (deg) & 0.370 & -0.879 & -0.242 & 0.13 \\
\hline & Standard deviation $(\hat{\sigma})$ & 0.414 & 0.357 & 0.301 & 0.21 \\
\hline \multirow[t]{2}{*}{ Steer angle variation } & Mean (deg) & 15.422 & 8.971 & 2.250 & 1.62 \\
\hline & Standard deviation $(\hat{\sigma})$ & 8.831 & 3.879 & 3.460 & 2.21 \\
\hline
\end{tabular}

Table 2 depicts the statistical results from conducting the ADVANCE-F automatic driving mode with the same running speed along the same roadway.

(1) Lateral displacement: The ADVANCE-F automatic steering system contrasts with human handling in the manner in which the vehicle behaves. The apparent lateral displacement when utilizing the automatic driving mode has a high correlation with vehicle speeds as higher speeds result in lower displacement $\left(\rho_{m}=0.9977\right)$. Also, the standard deviation has a tendency to decrease when speed increases ( $\left.\rho_{s}=-0.9963\right)$. In other words, the vehicle gains stability as it gains speed. This indicates that lane width and roadway clearance can be reduced when a roadway such as the Automated Highway Systems (AHS) is designed for high speeds with equipped vehicles.

(2) Yaw angle variation: The yaw angle is independent of speed $\left(\rho_{m}=-0.0197\right)$. However, the yaw angle variation and standard deviation gradually converge as the speed is increased $\left(\rho_{s}=-0.9922\right)$. This indicates that the automatic steering mode is capable of overcoming the vehicle's original deficiencies in body dynamics due to unbalanced loading, missing alignment, or photosensor' disposition/distortion.
(3) Steering angle variation: The steering angle remains positive regardless of the speed, proving that the testing vehicle has biased body dynamics. However, both the mean value and the standard deviation of the steering variation are reduced as the speed increases $\left(\rho=-0.9576, \rho_{s}=-0.8993\right)$. Consequently, the equipped vehicle is reliable in highspeed situations when in the automatic mode.

When comparing Table 2 with Table 1, most automatic mode values at speeds $40-50 \mathrm{kph}$ are smaller than those of manual driving. This fact indicates that the automatic mode is better than manual driving, at higher speeds. Although the automatic mode remains steady throughout the test, since its lower speed variations are somehow larger than that of manual driving, manual driving is still required to improve control.

\section{Meandering course}

In order to evaluate the controller's capability, the field test course should include the following: (a) switching the control from manual to automatic steering, (b) side wind disturbance, and (c) transition from a straight road into a curve. Thus, a meandering 
road section located on a southeastern mountain in Taiwan was chosen for the experiment. Around 13 curvature layouts with radii varying from 30 to $100 \mathrm{~m}$ are in the approximately $1.3 \mathrm{~km}$ road segment. There is also a $2 \%$ descending slope between the second curve and the fifth curve. The speed during the test varied from 20 to $50 \mathrm{kph}$. The testing day was sunny. Variations in lateral displacement, yaw angle differential, and steering angle differential were recorded. When the vehicle engaged the automatic driving mode its speed was around $26 \mathrm{kph}$. The vehicle then accelerated for $30 \mathrm{~s}$. Between 30 and $75 \mathrm{~s}$, its speed fluctuated between 33 and $36 \mathrm{kph}$. From the $75 \mathrm{th}$ second, the vehicle accelerated again. When vehicle speed achieved $44 \mathrm{kph}$, it then decelerated. The termination was at $120 \mathrm{~s}$. Based on the types of steering angle variation shown in Fig. 3, there are eight right-turn curves and five left-turn curves. As a group, their yaw angles were confined within the range of $-1.3^{\circ} \sim 2.5^{\circ}$ and the observed lateral deviations were smaller than $12 \mathrm{~cm}$. A standard lane width in Taiwan is $365 \mathrm{~cm}$ (12 feet, the same as in the USA). A passenger car is about $210 \mathrm{~cm}$ wide. Thus, the tolerance limit for an equipped passenger car's lateral displacement is $75.5 \mathrm{~cm}$ (i.e. $[365-210] / 2$ ). This indicates that the presented control was rather precise since the maximal lateral displacement in the test is only $12 \mathrm{~cm}$, smaller then the tolerance limit.

Table 3 provides further information about the test. According to the variation of steering angles in
Fig. 3, the test can be divided into 21 stages. Stage duration time, approximate speed, steering angle range, yaw angle range, and lateral deviation ranges are listed by stage in Table 3. Vehicle tracking is evaluated using steering angle range, yaw angle range, and lateral deviation range. It is evident that the steering angle values were opposite to that of lateral deviations when the vehicle encountered curves 1-7. This indicates that while the vehicle is turning right, its CG was located at the left-hand side with respect to the central line of the occupied lane, and vice versa for left-hand turns. During these stages, the yaw angle values were consistent with the lateral deviations.

The tracking control of the ADVANCE-F vehicle is clearly conservative in driving, there was no overshooting and it was stable. But at stage 8 , the compound curve forms a sharp but short left-turn curve followed by a smooth right-turn curve. In Fig. 3, due to speed increments at that moment, the steering quickly turned left to overcome the sudden negative lateral displacement. No sooner had the vehicle turned left then the yaw angle became positive due to the vehicle's encountering a right-turn curve. The steering quickly recovered and turned right until the yaw angle and lateral displacement tended to zero.

At stages 15-18, the vehicle encountered a much more complicated section of the road. The vehicle speed was also significantly increasing. When encountering
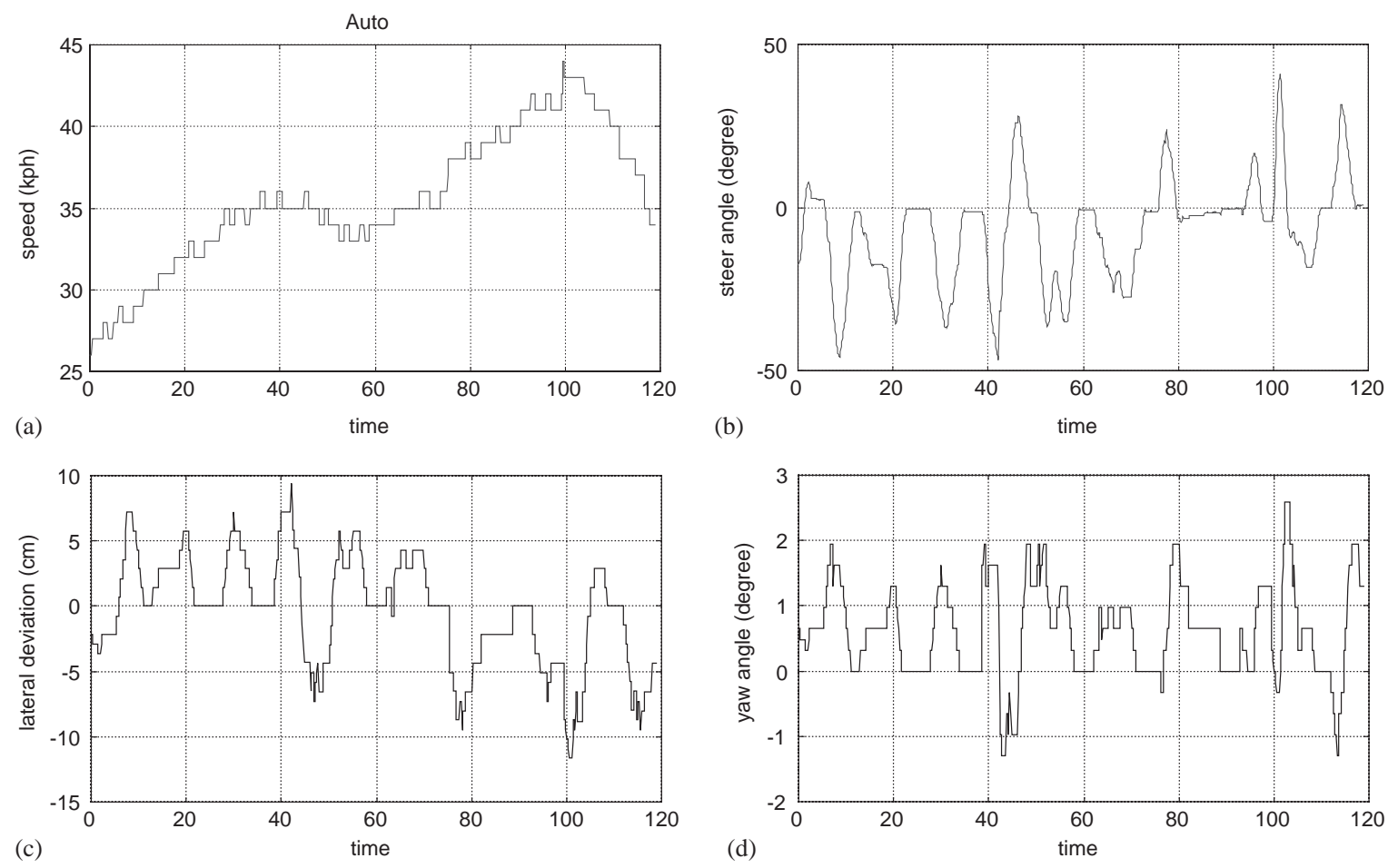

Fig. 3. (a-d) Results of the meandering course. 
Table 3

Information of the meandering course

\begin{tabular}{|c|c|c|c|c|c|c|}
\hline Stage & Duration (s) & $\begin{array}{l}\text { Road } \\
\text { condition }\end{array}$ & $\begin{array}{l}\text { Appx. speed } \\
(\mathrm{kph})\end{array}$ & $\begin{array}{l}\text { Steering angle } \\
\text { range (deg.) }\end{array}$ & $\begin{array}{l}\text { Yaw angle } \\
\text { range (deg.) }\end{array}$ & $\begin{array}{l}\text { Lateral deviation } \\
\text { range }(\mathrm{cm})\end{array}$ \\
\hline 1 & $0-5$ & $\sim$ straight & 27 & a & a & a \\
\hline 2 & $5-12$ & Curve $1, \mathrm{RT}$ & 29 & $-45 \sim 0$ & $0 \sim 2.0$ & $0 \sim 7.5$ \\
\hline 3 & $12-22.5$ & Curve 2,RT & 31 & $-35 \sim 0$ & $0 \sim 1.4$ & $0 \sim 6.0$ \\
\hline 4 & $22.5-27.5$ & Straight & 34 & 0 & 0 & 0 \\
\hline 5 & $27.5-35$ & Curve $3, \mathrm{RT}$ & 34.5 & $-35 \sim 0$ & $0 \sim 1.5$ & $0 \sim 7.5$ \\
\hline 6 & $35-39$ & Straight & 35.5 & 0 & 0 & 0 \\
\hline 7 & $39-42.5$ & Curve $4, \mathrm{RT}$ & 35 & $-45 \sim 0$ & $0 \sim 2.0$ & $0-9.0$ \\
\hline 8 & $42.5-49$ & Curve 5,LT & 34.5 & $0-30$ & $-1.3 \sim 0$ & $-7.0 \sim 0$ \\
\hline 9 & $49-59$ & Curve 6,RT & 34 & $-35 \sim 0$ & $0 \sim 2.0$ & $0 \sim 6.0$ \\
\hline 10 & $59-62.5$ & Straight & 34 & 0 & 0 & 0 \\
\hline 11 & $62.5-72.5$ & Curve 7,RT & 35.5 & $-30 \sim 0$ & $0 \sim 1.0$ & $0 \sim 4.0$ \\
\hline 12 & $72.5-76$ & Straight & 36 & 0 & 0 & 0 \\
\hline 13 & $76-89$ & Curve $8, \mathrm{LT} \sim \mathrm{RT}$ & 38.5 & $-5 \sim 25$ & $-0.3 \sim 2.0$ & $-9.0 \sim 0$ \\
\hline 14 & $89-93$ & $\sim$ straight & 39 & 0 & 0 & 0 \\
\hline 15 & $93-98$ & Curve 9,LT & 41.5 & $0 \sim 15$ & $0 \sim 0.7$ & $-7.5 \sim 0$ \\
\hline 16 & $98-100$ & Curve $10, \mathrm{RT}$ & 41.5 & $-3 \sim 0$ & $0 \sim 1.4$ & -5.0 \\
\hline 17 & $100-102$ & Curve $11, \mathrm{LT}$ & 42.5 & $0-40$ & $-0.3 \sim 0$ & $-12.0 \sim-5$ \\
\hline 18 & $102 \sim 110$ & Curve $12, \mathrm{RT}$ & 40 & $-20 \sim 0$ & $0 \sim 2.5$ & $-5 \sim 3.0$ \\
\hline 19 & $110-112.5$ & Straight & 37 & 0 & 0 & 0 \\
\hline 20 & $112.5-117$ & Curve $13, \mathrm{LT}$ & 35 & $0 \sim 30$ & $-1.3 \sim 0$ & $-10.0 \sim 0$ \\
\hline 21 & $117-120$ & Straight & 34 & $\mathrm{a}$ & a & $\mathrm{a}$ \\
\hline
\end{tabular}

Note: RT, Right turn; LT, Left turn.

${ }^{\mathrm{a}}$ Initial and final states.

curve 9, the lateral displacement was becoming increasingly negative such that the steering turned left. Nevertheless, there existed a slightly positive yaw angle. This short turn increased the yaw angle, because the vehicle encountered curve 10. Thus the steering turned back and then forward to the right. The situation during curves 11 and 12 is similar to curve 8 . The equipped vehicle was shaky from stages 15 to 18 , in that its speed was too fast to maneuver well. Finally, the vehicle arrived at stages 19 and 20 normally and then terminated at stage 21 . Apparently the controller is progressively successful on such a complicated road. Again, the lateral displacement is confined within $12 \mathrm{~cm}$, matching the criterion. This confirms that the controller is robust.

The test was performed at night. There is no affect on the automatic driving at daytime or at night since the marking detection system is composed of infrared equipment.

\section{Manual/auto switching process}

The ADVANCE-F's lateral control has modes for manual driving and automatic driving. The major control model for automatic driving is generally satisfactory as described above. However, switching in and out of the automatic mode is indeed very critical for control convergence, especially when switching from manual to automatic driving mode. Although the automatic steering system possesses highly intelligent equipment that can make optimal decisions when adapting to various circumstances, it is not as clever as a human being. Manual driving cannot be completely replaced by automatic driving and switching between the manual and automatic driving modes should be dependent upon the driver's will. The switching action should also be simple, quick, and safe.

When the equipped vehicle is running on a lane marked with special materials and the driver recognizes all conditions as suitable for automatic driving, he can press the "AUTO" button. The onboard computer will instantaneously check whether the marking sensors mounted under the front and real bumpers capture the guidance marking. It will indicate whether markings are captured by the photosensors and will actuate the automatic steering system when ready. Establishing a necessary delay time allows it to avoid over-shooting phenomenon. The onboard computer will continuously evaluate the control gain and will trigger the automatic steering mode when the gain reaches a smaller level than that at AUTO initiation. It generally needs at least 3-4s delay to resume control from manual handling after "yes" is reached. If "no", the automatic steering mode will not be actuated and a beeper or alarm will broadcast continuously. The driver should adjust the steering wheel until "yes" is reached, but can release his hands once the automatic mode is actuated. 
Switching from automatic to manual driving mode is simpler than from manual to automatic. The driver merely has to touch the "MANUAL" key and it transfers without any delay. The auto mode will sound an alarm and then release control if the following situations occur (Chang, 1996b):

(1) Reaching the end of the special lane: The computer will remind the driver to switch the driving mode or the security system will light up a warning lamp and sound an alarm when the vehicle reaches the end of the special material-marked lane.

(2) Component breakdown: The system will immediately light up the warning lamp and sound an alarm to remind the driver to switch the driving mode if it detects that any components are out of order.

(3) Marking failure: The controller will send out a detection error message and also trigger the warning light and alarm when the marking is blurry or broken.

(4) Lane-changing: The driver must switch to the manual driving mode if he/she wants to change lanes since the system is not yet capable of automatically changing lanes.

(5) Emergency: The driver should switch to manual mode if there is an obstacle or some other irregularity in the lane ahead.

(6) Weather effect: Bad weather may seriously affect the accuracy of the system. Warning devices notify the driver to switch back to the manual driving mode when strong winds or storms occur.

\section{Conclusions}

The ADVANCE-F automatic steering control model is almost complete. According to the preliminary tests on the meandering course, the controller is robust and satisfactory. The test comparison also reveals that human driving is quite different from automatic driving. The lateral deviation of human driving is independent of vehicle speeds whereas that of automatic driving is dependent on vehicle speeds. The automatic driving mode can overcome a vehicle's original deficiencies in body dynamics as a result of unbalanced loading, missing alignment or photosensors' disposition/distortion. The automatic mode seems to be reliable in high speeds as the vehicle actually becomes more stable in high-speed situations. However, the test was not conducted at a speed beyond $50 \mathrm{kph}$. Also, compared to an experienced driver, the automatic mode is still somehow unsteady at low speeds. The greatest weakness of the study is only providing one meandering course in a single field, $1.3 \mathrm{~km}$ long. Such deficiencies are to be improved in future work.

The field test reveals that the manner of switching in and out of the AUTO mode is critical for continuous control and safety. The algorithms contained herein included: (1) from the manual driving mode to the automatic driving mode and (2) from the automatic driving mode to the manual driving mode. The main feature of the system is that the automatic steering mode is triggered as when the control gain reaches a lower level. The driver will feel comfortable because the handoff needs at least a 3-4s delay before the machine fully takes control, to avoid the mechanical overshooting phenomenon.

\section{References}

Chang, T-H. (1993). Study and experiment on vehicle control of an automated highway system ADVANCE-F. Technical Report of the Institute of Transportation, MOTC, Taiwan, ROC, No. 82-39-617 (in Chinese).

Chang, T-H. (1995). Modeling and comparison of man-machine driving on an ADVANCE-F Vehicle. Technical Report of the National Science Council, Taiwan, ROC, No. NSC 83-0410-E-032-014, No. NSC 84-0410-E-032-004 (in Chinese).

Chang, T-H. (1996a). Vehicle lateral control modeling for steering automation in the ADVANCE-F system. Transactions of the Institute of Measurement and Control, 18(3), 149-159.

Chang, T-H. (1996b). Initial control algorithm for switching-in and switching-out an automatic steering system: ADVANCE-F. Proceedings of the $3 r d$ World Congress on ITS, ITS America.

Fenton, R. E. (1979). A headway safety policy for automated highway operations. IEEE Transactions on Vehicular Technology, VT-28(1), $22-28$.

Fenton, R. E. (1988). On the optimal design of an automotive lateral controller. IEEE Transaction on Vehicular Technology, 37(2), $108-113$.

Ioannou, P. A., Chien, C. C., \& Hauser, J. (1992). Autonomous intelligent cruise control. Proceedings of the IVHS America 1992 annual meeting. Vol. 1, pp. 97-112.

Kuo, B. C. (1991). Automatic control systems (6th ed.). Englewood Cliffs, NJ: Prentice-Hall.

Rao, B. S. Y., Varaiya, P., \& Eskafi, F. (1993). Investigations into achievable capacities and stream stability with coordinated intelligent vehicles. TRB 72nd Annual Meeting. Washington, DC, Paper No. 930803.

Vegte, J. V. D. (1994). Feedback control systems (3rd ed.). Englewood Cliffs, NJ: Prentice-Hall International Inc. 